OPEN ACCESS

Edited by:

Smita S. Iyer,

Emory University, USA

Reviewed by:

Mette D. Hazenberg,

University of Amsterdam,

Netherlands

Guido Ferrari,

Duke University, USA

${ }^{*}$ Correspondence:

John Frater

john.frater@ndm.ox.ac.uk

tThese authors have contributed equally to this work.

Specialty section: This article was submitted to HIV and AIDS,

a section of the journal

Frontiers in Immunology

Received: 05 October 2016 Accepted: 11 January 2017

Published: 30 January 2017

Citation:

Thornhill JP, Fidler S, Klenerman P, Frater $J$ and Phetsouphanh C (2017) The Role of CD4+ T Follicular Helper

Cells in HIV Infection: From the Germinal Center to the Periphery.

Front. Immunol. 8:46.

doi: 10.3389/fimmu.2017.00046

\section{The Role of CD4+ T Follicular Helper Cells in HIV Infection: From the Germinal Center to the Periphery}

\author{
John Patrick Thornhill', Sarah Fidler ${ }^{1}$, Paul Klenerman ${ }^{2}$, John Frater ${ }^{2 *}$ and \\ Chansavath Phetsouphanh ${ }^{2 \dagger}$
}

${ }^{1}$ Imperial College London, London, UK, ${ }^{2}$ University of Oxford, Oxford, UK

T follicular helper cells (TFh) are key components of the adaptive immune system; they are primarily found in germinal centers (GCs) where their interaction with B cells supports humoral immune responses and efficient antibody production. They are defined by the expression of CXC receptor 5, program death-1, ICOS, and secretion of IL-21. Their differentiation is regulated by B-cell lymphoma 6 . The relationship and function of circulating TFh to bona fide TFh resident in the GC is much debated. HIV infection impacts the TFh response with evidence of aberrant TFh function observed in acute and chronic infection. Effective TFh responses are associated with the development of broadly neutralizing antibody responses to HIV and may be important for viral control. In addition, TFh are preferentially infected and act as a key reservoir for latent HIV infection. This review explores recent developments in our understanding of TFh differentiation, regulation, function, and the relationship between cTFh and those in GCs, and the complex interaction between TFh and HIV infection.

Keywords: HIV, Tfh cells, reservoir, T cells, viral immunity

\section{INTRODUCTION}

Optimal immune function requires effective communication between all arms of the immune system. An efficient humoral immune response to pathogens is dependent on the interaction between helper $\mathrm{T}$ cells and $\mathrm{B}$ cells. T cell help is critical to optimize antibody production and class switching and allows for the development and production of targeted high-affinity antibodies. The subset of $\mathrm{T}$ helper cells responsible for this interaction remained elusive until the beginning of this century when Kim et al. described a subset of CXC receptor 5 (CXCR5)+ T cells in the germinal center (GC) with B helper activity (1) coined T follicular helper cells (TFh). TFh cells play a significant role in supporting B cell activation and antibody production during the humoral immune response. They are critical for B cell support, somatic hypermutation (SHM), and antibody class switching (2). TFh dysfunction and expansion has been implicated in a number of disease states including Rheumatoid Arthritis (3), SLE (4), and other autoimmune diseases.

In health, the interaction between Tfh, B cells, and IL-21 supports B cells to proliferate and differentiate into plasma cells thereby leading to efficient antibody production. Consequently, the relationship between TFh cells, humoral immunity, and mechanisms for viral persistence in the setting of chronic viral infections has received much attention. The role of TFh in supporting specific antibody responses has been described in hepatitis C (5), hepatitis B (6), and lymphocytic choriomeningitis virus (LCMV) clearance in mice (7). In acute hepatitis C, the development of HCV-specific 
antibodies correlates with increased ICOS expression (5), while increased frequencies of TFh is associated with an active Hepatitis B phenotype (6). In the murine model, epigenetic changes which support TFh differentiation have been shown to be important for resolution of LCMV infections (7). These observations support the role of TFh cells in the development of effective pathogenspecific antibody responses to chronic viral infections. The interplay between HIV infection and TFh cells is complex, with evidence supporting dysregulation of TFh function by HIV itself and conversely, that the frequency of TFh subsets positively correlates with effective humoral responses to HIV, as measured by the development broadly neutralizing antibodies (BNAbs) (8). This review aims to summarize the current data and recent advances in our understanding of the role of TFh in HIV infection, with particular emphasis on the phenotypic and functional differences between GC and peripheral-circulating TFh cells (pTFh).

\section{PHENOTYPIC AND TRANSCRIPTIONAL PROFILE OF TFh CELLS}

$\mathrm{T}$ follicular helper cells are a subset of memory CD4 T cells that are localized in the B-cell follicles of secondary lymphoid tissues and provide help for B cells. TFh-B cell interactions allow the production of high affinity, class-switched antibodies following natural infection or vaccination, as well as the establishment of B cell memory (9). TFh cells are defined by their expression of high levels of surface markers of program death-1 (PD-1) and chemokine CXCR5, especially within the GCs of secondary lymphoid tissue. PD-1+ CXCR5+ phenotypically distinguishes GC T-follicular helper cells from other T helper cell subsets and from peripheral CXCR5 + cells with the capacity for B cell help (Figure 1A). This phenotype holds true for GC TFh cells, but peripheral-circulating TFh cells seem to have lower levels of PD-1, where a considerable proportion of CD4+ T cells express only CXCR5 (Figure 1B). However, TFh cells' identity as a "bona fide" subset of T helper cells was not established until B-cell lymphoma 6 (Bcl-6) was discovered to be the "master" transcription factor that drives TFh cell differentiation and function (10-12).

In the early 2000s, several groups described CXCR5+ T cells as having the preferential ability to activate $\mathrm{B}$ cells to produce classswitched antibodies $(1,13)$. The chemokine receptor CXCR5 plays an important role in promoting $\mathrm{T}$ cell and $\mathrm{B}$ cell migration into $\mathrm{B}$ cell follicles in response to its interactions with CXCL13 (14). It has since been shown that these CXCR5+ T cells are TFh cells with a unique gene-expression profile compared to other CD4 T cell subsets. TFh cells do not express Tbet, GATA3, RORyt, or Foxp3 and produce limited Th1/Th2/Th17 related cytokines. Gene-expression profiling of CXCR5+ TFh cells identified key molecules, i.e., BCL6, ASCL2, IL21, PDCD1 (PD-1), and ICOS, all of which are involved in the development, migration, and function of these cells $(15,16)$.

Naive CD4 T cell differentiation toward the TFh lineage is primed by antigen-presenting dendritic cells (DCs) in the T cell zone, through the interaction between $\mathrm{T}$ cell receptor and major histocompatibility complex class II, and costimulatory molecules CD28 and ICOS. Activated antigen-specific CD4 T cells downregulate CCR7 and upregulate CXCR5, allowing them to migrate to $\mathrm{B}$ cell follicles. It has recently been shown that the initial step in TFh induction is the upregulation of aschaete-scute homolog 2 (Ascl2), which is a transcription factor that can induce CXCR5 expression, enabling pre-TFh cells to migrate toward the border of the B-cell follicle. Ascl2 has also been shown to repress non-TFh genes, allowing pre-TFh to differentiate into the follicular pathway (17).

Dendritic cells and B cells collaborate in TFh induction in a sequential manner, whereby TFh priming by DCs occurs prior to $\mathrm{B}$ cell presentation, allowing for optimal TFh development and GC formation $(18,19)$. DCs also secrete cytokines IL-6 and IL-12, which induces an early wave of Bcl-6 expression in pre-TFh cells, in a STAT3-dependent manner $(20,21)$. pre-TFh cells that receives a second signal from cognate antigen-primed B cells stabilizes Bcl-6 express (22). Bcl-6 corroborates with other transcription factors such as, IRF4, BATF and c-Maf, to drive the expression of Tfh cell signature genes critical for T cell-B cell interaction, which includes CXCR5, ICOS, Sh2d1a Pdcd1, and CD40L (11). IL-27 can induce c-maf expression in collaboration with $\operatorname{ICOS}(23,24)$. C-maf induces IL-21 production in CD4 T cells committed to TFh pathway. IL-21 acts as an autocrine cytokine to promote pre-TFh cell differentiation and homeostatic maintenance of TFh cells. It also plays a vital role in differentiation of GC B cells into memory and plasma cells. Optimal interaction between TFh and $B$ cells determines the magnitude of the GC reaction and somatic mutation that in turn controls affinity maturation of B cells and, therefore, the breadth of the antibody response (25-27).

\section{PERIPHERAL-CIRCULATING AND GC TFh CELLS}

CXC receptor $5+$ circulating TFh ( $\mathrm{pTFh}$ )-like cells can also be found in human peripheral blood. However, the phenotype of pTFh is not as "clear-cut" compared to GC TFh. They are generally defined as being CXCR5+, CCR7lo, PD-1+, and ICOS+, although this phenotype is not as stringent as GC TFh (28). Others have reported pTFh to co-express CCR7 and are included in the central memory subset. It has also been shown that CXCR5+ Tcm cells lack CXCR3 and CCR4 and do not differentiate into Th1 or Th2 cells upon polarizing cytokine stimulation $(2,29)$. CXCR5 and PD-1 are stably expressed on pTFh cells and are not transiently upregulated upon non-specific antigen or cytokine stimulation. pTFh expressing these markers can help B-cells to differentiate into plasmablasts, but require secondary signals from B-cells, such as CD40L or ICOS interactions, as well as IL-21 secretion $(8,30,31)$. However, blood pTFh and lymphoid TFh cells are clearly phenotypically different, particularly with respect to the expression of PD-1 and BCL-6 (32). Bcl-6 expression can be used to determine TFh in GCs (Figure 1B), but this is not the case in peripheral blood (Figure 1D), where Bcl-6 seem to be downregulated in ex vivo CD4 T cells. CXCR5+ PD-1+ pTFh subset contains higher IL21 mRNA compared to the other CD4+ subsets (Figure 1C) (unpublished data), with CXCR5+ PD-1- subset expressing higher levels of ICOS. It remains to be seen whether the CXCR5+ PD-1- subset are precursors to GC 

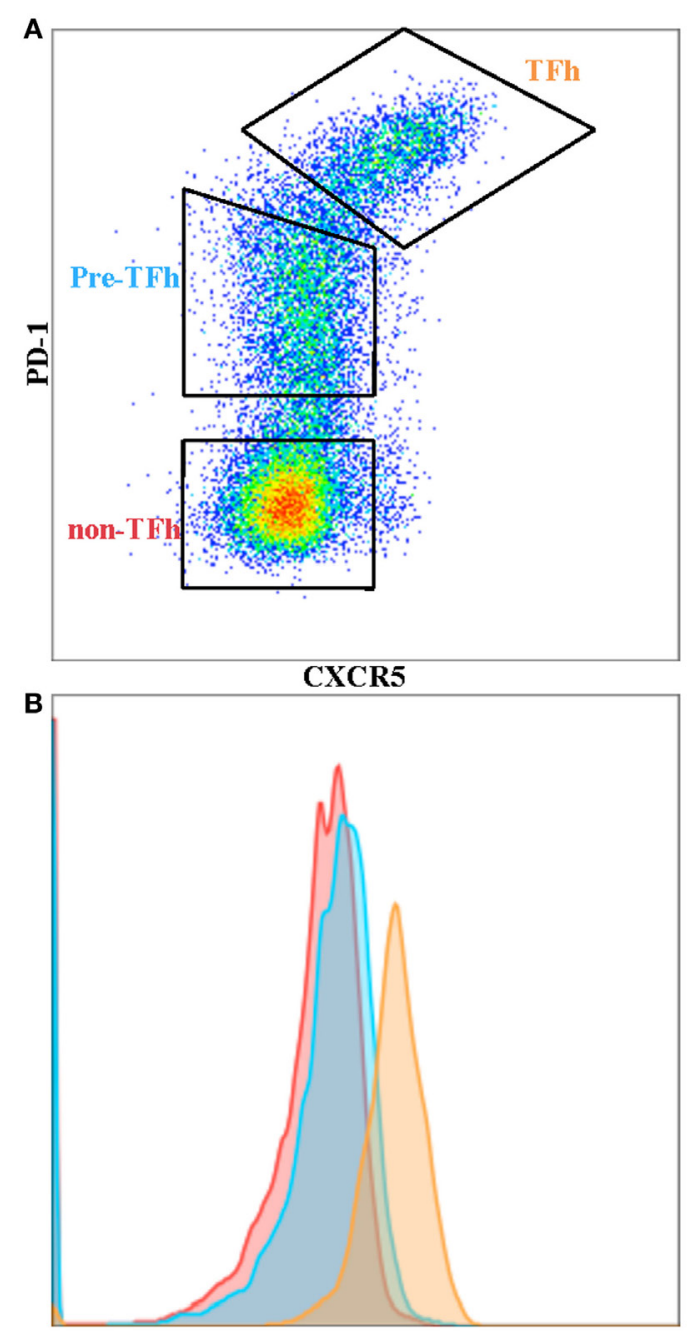

BCL-6

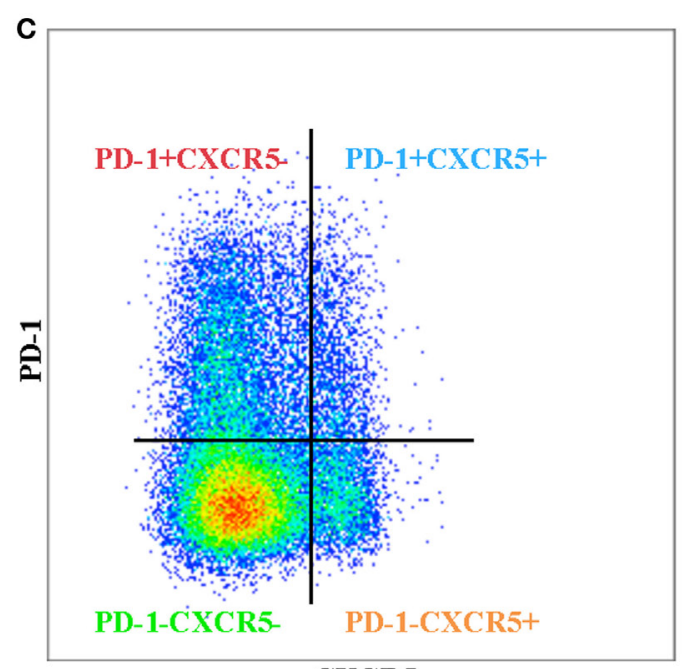

CXCR5

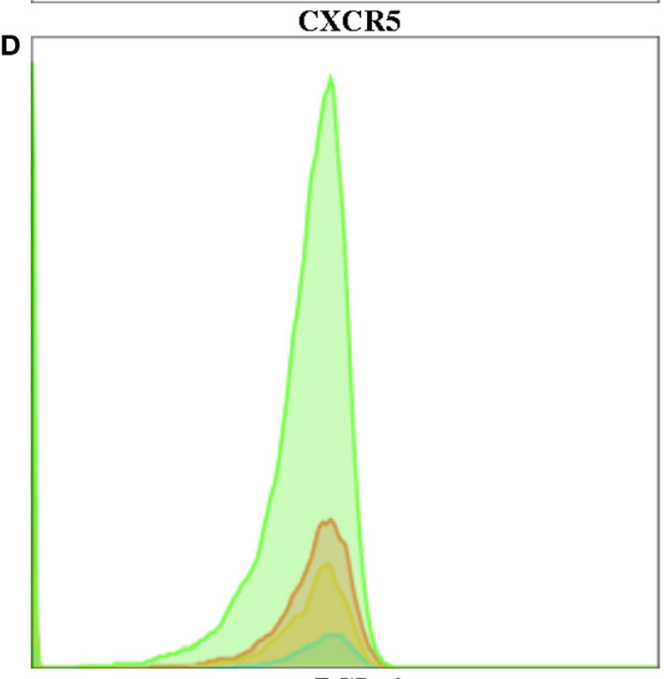

BCL-6

FIGURE 1 | Phenotypic differences between tonsil and peripheral blood T follicular helper cells (TFh). (A) TFh, pre-Tfh, and non-TFh subsets in human tonsil. (B) BCL-6 expression on TFh (orange), pre-TFh (blue), and non-TFh (red) subsets in tonsil. (C) Ex vivo peripheral blood CD4 T cells separated into four quadrants comprising program death-1 (PD-1) - CXC receptor 5 (CXCR5)-, PD-1+ CXCR5-, PD-1+ CXCR5+ and PD-1- CXCR5+ subsets. (D) BCL-6 expression on PD-1 - CXCR5- (green), PD-1+ CXCR5- (red), PD-1+ CXCR5+ (blue), and PD-1-CXCR5+ (orange) subsets in peripheral blood.

TFh or TFh cells that have lost PD-1 expression in the periphery. Iyer and colleagues have shown that blood TFh may be a potential surrogate for GC responses by using a Rhesus macaque model (33). They sampled blood and lymph node at 1 and 2 weeks following SIV booster immunization and demonstrated that blood TFh effector responses (identified by Ki-67+ CXCR5+ expression) predicted magnitude of subsequent GC responses. Further studies in different disease contexts are required to correlate the phenotypic and functional differences previously observed between pTFh and GC TFh in order to understand the important dynamics of this subset in blood and tissue.

\section{HIV AND TFh DYSFUNCTION}

The impact of HIV on the frequency and function of TFh subsets during both treated and untreated HIV infection is poorly understood. Host, viral, and treatment factors, such as the timing of Antiretroviral Therapy (ART) initiation with respect to stage of HIV infection duration, may influence the ability of TFh cells to support effective humoral responses. Furthermore, the significance of pTFh and their relationship to GC TFh cells is much debated in health (34) and is likely to be further complicated by chronic HIV infection.

Untreated chronic HIV infection results in selective accumulation of TFh cells in lymph nodes (35); Lindqvist et al. have demonstrated the relative accumulation of HIV-specific TFh cells in lymph nodes during chronic untreated HIV infection that was associated with HIV viremia, interestingly the majority of the expanded TFh cells were specific for Gag, as opposed to Env (36). The expansion of TFh is in contrast to the expected depletion of activated total CD4 T cells seen in untreated HIV infection. The observed TFh expansion is thought to be driven 
by persistent antigenic stimulation mediated in part by IL-6 signaling (37) which in turn drives the abnormal expansion of TFh cells, skewed B cell differentiation and impaired antibody production (35). Work by Cubas et al. support these findings and suggest PD-1 triggering by PD-L1 on Germial Center B cells as a mechanism for the observed abberant TFh dysfunction (38); their work demonstrates that PD-1 blockade enhances HIV-specific immunoglobulin production in vitro and that triggering of PD-1 on TFh reduces cell proliferation and activation, in addition to a decrease in IL-21 production (38). IL-21 has been shown to be essential for provision of B cell help by TFh (30), and its addition rescues antibody production in vitro (37). More recent evidence suggest that the loss of regulatory control by $\mathrm{T}$ follicular regulatory (TFR) cells may also play a role in the inefficient GC responses seen in untreated HIV infection (39). Data from Rhesus Macques (RM) indicate that TFR may contribute to the regulation of TFh and GC B cells and that decreases in the TFR/TFh ratio during chronic SIV infection may lead to an uncontrolled expansion of both TFh and GC B cells (40).

The difficultly in sampling the GC TFh population has prompted investigation of $\mathrm{pTfh}$ as a surrogate marker for GC Tfh activity. Studies have demonstrated the ability of $\mathrm{pTfh}$ to provide B cell help $(30,41,42)$. Work by Boswell et al. showed differential cytokine production by pTFh (such as IL-21, IL-2, and IL-17) compared with germinal Tfh (IL-4, IL-10, and IL-21, but compromised production of IL-2 and IL-17) (41), while Locci et al. have found that PD-1+ CXCR3- CXCR5+ CD4+ T cells were the subset with greatest ability to provide $\mathrm{B}$ cell help (8). Contrary to the expansion of GC TFh seen in chronic HIV, a study of pTFh reported a significant decrease in the $\mathrm{pTFh}$ population from HIVinfected subjects compared to HIV-uninfected subjects, however this study did not involve concurrent sampling of GC Tfh (41). In addition, pTfh of chronic ART-treated aviremic individuals have been found to be functionally impaired in their ability to provide adequate $B$ cell help when compared with those from elite controllers (43), while vaccine-induced $\mathrm{Ab}$ response to influenza has been associated with preserved Tfh function as measured by the secretion of IL-21 and CXCL13 (44). A recent study by Schultz et al. used an IL-21 capture assay to identify circulating IL-21+ CD4+ T cells. These were found to be transcriptionally and phenotypically pTFh cells (45). In addition to B cell help, IL-21 signaling also supports the antiviral function of CD8 and NK cells thereby playing an important role in control of chronic viral infections $(46,47)$. Increases in IL-21-producing blood CD4+ T cells have been observed during acute and chronic HIV infection with the elevated frequencies of HIV-specific IL-21producing CD4+ $\mathrm{T}$ cells associated with viral control (48). An expansion of HIV-specific IL-21+ pTFh cells in the ALVAC+ AIDSVAX vaccine study supports the hypothesis that induction of TFh cells might be involved in the superior humoral response (45). Taken together, these data seem to suggest that, in some individuals, HIV infection depletes pTFh numbers and impairs their function, while those better able to maintain robust TFh functionality may have greater propensity for viral control; however, lack of a standardized phenotypic definition for pTFh may confuse some of the reported findings. Furthermore, TFh function may be impacted by ART; initiation of ART early or during
Primary HIV infection has been shown to be associated with later posttreatment viral control (49); a recent study investigated the impact of ART in acute HIV infection on TFh function report that early ART may prevent immune dysregulation while preserving pTFh function and B-cell memory, however, the benefit was less if ART is commenced in later stages of acute infection (36).

\section{TFh, BROADLY NEUTRALIZING ANTIBODIES, AND HIV CONTROL}

Hypergammaglobulinemia is a feature of untreated HIV infection and has been linked to an aberrant TFh-B cell interaction $(37,50)$, studies have shown a correlation between the expression of Bcl-6 in TFh cells and levels of total serum IgG antibody levels in untreated HIV (36). In the LCMV model, a model for chronic viral infection, the expanded TFh population has been shown to activate non-specific B cells resulting in hypergammaglobulinemia. This has also been observed during other persistent viral infections including HIV and hepatitis C (51).

The generation of BNAbs against HIV is observed in up to $20 \%$ of HIV-positive individuals (52). BNAbs are characterized by high levels of SHM which is supported by TFh during the GC reaction (53). Cohen et al. have shown an association between the frequency of PD-1+ CXCR5+ CD4+ Tfh cells during early-untreated HIV infection and future BNAbs development (54). A separate study has shown that the frequency of pTFh cells or more specifically PD-1+ CXCR3 - CXCR5 + CD4+ T cells correlate with broadly neutralizing antibody responses (5). A longitudinal study using RMs found that high levels of continuous Env antigen production are required for driving GC TFh activation, which in turn leads to more effective broadly neutralizing antibody responses (55). In that study, Env-specific TFh cells but not total TFh correlated with IgG+ GC B cells and effective antibody production (55). These data imply that a period of uncontrolled viral replication (or adequate vaccination) may be required for a sufficient level of antigen exposure and subsequent effective BNAbs production.

The role of immune tolerance has also been implicated in the development of BNAbs, work by Moody et al., show that HIV-1infected individuals with BNAbs had a higher frequency of blood autoantibodies, a lower frequency of regulatory CD4+ T cells, a higher frequency of memory pTFh, and a higher TFR PD-1 expression compared with HIV-1-infected individuals without BNAbs. The authors suggest the balance of TFR to TFh may allow for development of BNAbs (56).

\section{TFh SUPPORT HIV PERSISTENCE}

Effective ART reduces plasma viral replication to undetectable levels (as measured by conventional HIV RNA qPCR assays to $<20$ copies HIV RNA/ml). However, complete eradication of HIV is still unachievable due to low-level viral replication in sanctuary sites and reactivation of virus from the latent HIV DNA reservoir. Suppression of HIV viremia in peripheral blood does not necessarily reflect viral suppression in lymphoid tissue with variable tissue penetration of ART (57), further potentially skewing the relationship between HIV and GC and pTFh. In chronic treated 
HIV infection, lymphoid tissue is the primary site of ongoing HIV replication $(58,59)$. GC TFh cells have been implicated in HIV persistence by supporting viral replication during treated infection and serving as an important cellular reservoir of HIV-1 DNA.

Germinal center TFh cells are highly permissive to HIV infection, with downregulation of PD-1 during HIV-1 replication (37). Cell sorting experiments by Perreau et al. have demonstrated that TFh and CXCR5- PD-1+ populations in lymph node are most efficient in supporting HIV replication (45). A recent study looked at the role of pTFh, defined phenotypically as CD45RA-CCR7+ CXCR5+, in HIV persistence, they used an in vitro GFP reporter assay and found pTFh, in particular, PD1+ pTFh cells to be more permissive for HIV infection than non-pTFh cells (60).

T Follicular helper cells cells demonstrate greater HIV viral production compared to other CD4+ T-cell subsets. In untreated HIV-1 infection, CXCR5+ CD4+ T-cell subsets have been shown to contain 11- to 66-fold more HIV-1 RNA than CXCR5- subsets (37). A study in chronic HIV infection of pTFh cells demonstrated greater HIV production (measured by p24 expression after antiCD3/anti-CD28 stimulation). Also, higher frequencies of 2-LTR circles were observed in the pTFh cells than in non-pTFh cells, confirming the idea that TFh cells support HIV persistence during ART-treated HIV infection (60).

Studies in non-human primates support the hypothesis that TFh cells in GCs act as a sanctuary site for ongoing viral replication in otherwise controlled SIV infection (61). Certain primates that can spontaneously control plasma SIV replication to levels below the limit of detection are termed "Elite" controlling monkeys, where HIV-specific CD8+-mediated viral control in extrafollicular sites was observed. Such monkeys had evidence of ongoing viral replication in GCs, from which HIV-specific CD8+ $\mathrm{T}$ cells are excluded (61). Bortitz et al. have published work that supports this mechanism of persistence; using samples from elite controllers, they detected viruses in lymph node with genetic and transcriptional markers of active replication most abundantly

\section{REFERENCES}

1. Kim CH, Rott LS, Clark-Lewis I, Campbell DJ, Wu L, Butcher EC. Subspecialization of CXCR5+ T cells: B helper activity is focused in a germinal center-localized subset of CXCR5+ T cells. J Exp Med (2001) 193:1373-81. doi:10.1084/jem.193.12.1373

2. Breitfeld D, Ohl L, Kremmer E, Ellwart J, Sallusto F, Lipp M, et al. Follicular B helper T cells express CXC chemokine receptor 5, localize to B cell follicles, and support immunoglobulin production. J Exp Med (2000) 192:1545-52. doi:10.1084/jem.192.11.1545

3. Wang J, Shan Y, Jiang Z, Feng J, Li C, Ma L, et al. High frequencies of activated $\mathrm{B}$ cells and $\mathrm{T}$ follicular helper cells are correlated with disease activity in patients with new-onset rheumatoid arthritis. Clin Exp Immunol (2013) 174:212-20. doi:10.1111/cei.12162

4. Zhang X, Lindwall E, Gauthier C, Lyman J, Spencer N, Alarakhia A, et al. Circulating CXCR5+CD4+helper T cells in systemic lupus erythematosus patients share phenotypic properties with germinal center follicular helper T cells and promote antibody production. Lupus (2015) 24:909-17. doi:10.1177/0961203314567750

5. Raziorrouh B, Sacher K, Tawar RG, Emmerich F, Neumann-Haefelin C, Baumert TF, et al. Virus-specific CD4+T cells have functional and phenotypic characteristics of follicular T-helper cells in patients with acute and chronic within PD1+, TFH-enriched cell populations (62). Recently, a specialized group of cytotoxic T cells that express CXCR5 have been described and termed "follicular cytotoxic T cells." These cytotoxic cells were shown to eradicate TFh-infected cells (63). A greater understanding of this subtype will be pivotal to eradicate latently infected GC TFh cells.

\section{CONCLUSION}

Strong antibody responses are pivotal to the eradication of many pathogenic infections. The importance of TFh cells in regulating B cell development and function to produce broad neutralizing antibodies is now clearly evident. TFh dysregulation in HIV infection has been well characterized. Whether these cells are preferentially infected within GCs and remain as latent reservoirs still requires further investigation. Also, whether Pre-TFh cells within secondary lymphoid tissue are targets of HIV due to their high expression of CCR5 and whether they are able to upregulate PD-1 and become fully functioning TFh cells is still unclear. Molecules targeting TFh cell function, such as PD-1 blocking antibodies and recombinant IL-21 administration are currently being used in therapeutic cancer trials. Results from these trials will determine if they would be beneficial as immunotherapy in HIV infection. However, a caveat of using such therapies in the context of HIV infection is that although it may improve TFh-B cell interactions and antibody production, immune modulation of TFh function may also increase $\mathrm{T}$ cell activation and lead to reactivation of the virus that may lead to an increase in additional reservoirs within GCs. Detailed studies of this important subset in terms of its function within the GCs and its relationship to other $\mathrm{T}$ cell and B cell subsets will shed light on possible therapeutics that may be useful in HIV infection.

\section{AUTHOR CONTRIBUTIONS}

JT wrote the manuscript. SF and PK edited the manuscript. JF and $\mathrm{CP}$ are equal contributors; both wrote and edited the manuscript.

HCV infections. Gastroenterology (2016) 150:696.e-706.e. doi:10.1053/j. gastro.2015.11.005

6. Feng J, Lu L, Hua C, Qin L, Zhao P, Wang J, et al. High frequency of CD4+ CXCR5+ TFH cells in patients with immune-active chronic hepatitis B. PLoS One (2011) 6:e21698. doi:10.1371/journal.pone.0021698

7. Cook KD, Shpargel KB, Starmer J, Whitfield-Larry F, Conley B, Allard DE, et al. Cell-dependent clearance of a persistent virus infection requires $\mathrm{T}$ cell expression of the histone demethylase UTX. Immunity (2015) 43:703-14. doi:10.1016/j.immuni.2015.09.002

8. Locci M, Havenar-Daughton C, Landais E, Wu J, Kroenke MA, Arlehamn CL, et al. Human circulating PD-1+CXCR3-CXCR5+ memory Tfh cells are highly functional and correlate with broadly neutralizing HIV antibody responses. Immunity (2013) 39:758-69. doi:10.1016/j.immuni.2013. 08.031

9. Crotty S. Follicular helper CD4 T cells TFH. Annu Rev Immunol (2011) 29:621-63. doi:10.1146/annurev-immunol-031210-101400

10. Yu D, Batten M, Mackay CR, King C. Lineage specification and heterogeneity of T follicular helper cells. Curr Opin Immunol (2009) 21:619-25. doi:10.1016/j. coi.2009.09.013

11. Choi YS, Yang JA, Crotty S. Dynamic regulation of Bcl6 in follicular helper CD4 T Tfh. cells. Curr Opin Immunol (2013) 25:366-72. doi:10.1016/j. coi.2013.04.003 
12. Johnston RJ, Poholek AC, DiToro D, Yusuf I, Eto D, Barnett B, et al. Bcl6 and Blimp-1 are reciprocal and antagonistic regulators of $\mathrm{T}$ follicular helper cell differentiation. Science (2009) 325:1006-10. doi:10.1126/science.1175870

13. Schaerli P, Willimann K, Lang AB, Lipp M, Loetscher P, Moser B. CXC chemokine receptor 5 expression defines follicular homing $\mathrm{T}$ cells with $\mathrm{B}$ cell helper function. J Exp Med (2000) 192:1553-62. doi:10.1084/jem.192.11.1553

14. Ansel KM, McHeyzer-Williams LJ, Ngo VN, McHeyzer-Williams MG, Cyster JG. In vivo-activated CD4 T cells upregulate CXC chemokine receptor 5 and reprogram their response to lymphoid chemokines. J Exp Med (1999) 190:1123-34. doi:10.1084/jem.190.8.1123

15. Kim CH, Lim HW, Kim JR, Rott L, Hillsamer P, Butcher EC. Unique gene expression program of human germinal center T helper cells. Blood (2004) 104:1952-60. doi:10.1182/blood-2004-03-1206

16. Vinuesa CG, Linterman MA, Yu D, MacLennan IC. Follicular helper T cells. Annu Rev Immunol (2016) 34:335-68. doi:10.1146/ annurev-immunol-041015-055605

17. Liu X, Chen X, Zhong B, Wang A, Wang X, Chu F, et al. Transcription factor achaete-scute homologue 2 initiates follicular T-helper-cell development. Nature (2014) 507:513-8. doi:10.1038/nature12910

18. Deenick EK, Chan A, Ma CS, Gatto D, Schwartzberg PL, Brink R, et al. Follicular helper $\mathrm{T}$ cell differentiation requires continuous antigen presentation that is independent of unique B cell signaling. Immunity (2010) 33:241-53. doi:10.1016/j.immuni.2010.07.015

19. Barnett LG, Simkins HM, Barnett BE, Korn LL, Johnson AL, Wherry EJ, et al. B cell antigen presentation in the initiation of follicular helper T cell and germinal center differentiation. J Immunol (2014) 192:3607-17. doi:10.4049/ jimmunol.1301284

20. Karnowski A, Chevrier S, Belz GT, Mount A, Emslie D, D’Costa K, et al. B and $\mathrm{T}$ cells collaborate in antiviral responses via IL-6, IL-21, and transcriptional activator and coactivator, Oct2 and OBF-1. J Exp Med (2012) 209:2049-64. doi:10.1084/jem.20111504

21. Ma CS, Suryani S, Avery DT, Chan A, Nanan R, Santner-Nanan B, et al. Early commitment of naive human $\mathrm{CD} 4+$. $\mathrm{T}$ cells to the $\mathrm{T}$ follicular helper TFH. cell lineage is induced by IL-12. Immunol Cell Biol (2009) 87:590-600. doi:10.1038/icb.2009.64

22. Wallin EF, Jolly EC, Suchanek O, Bradley JA, Espeli M, Jayne DR, et al. Human T-follicular helper and T-follicular regulatory cell maintenance is independent of germinal centers. Blood (2014) 124:2666-74. doi:10.1182/ blood-2014-07-585976

23. Batten M, Ramamoorthi N, Kljavin NM, Ma CS, Cox JH, Dengler HS, et al. IL-27 supports germinal center function by enhancing IL-21 production and the function of T follicular helper cells. J Exp Med (2010) 207:2895-906. doi:10.1084/jem.20100064

24. Bauquet AT, Jin H, Paterson AM, Mitsdoerffer M, Ho IC, Sharpe AH, et al. The costimulatory molecule ICOS regulates the expression of c-Maf and IL-21 in the development of follicular T helper cells and TH-17 cells. Nat Immunol (2009) 10:167-75. doi:10.1038/ni.1690

25. Sallusto F. Heterogeneity of human CD4+. T cells against microbes. Annu Rev Immunol (2016) 34:317-34. doi:10.1146/annurev-immunol-032414-112056

26. Chtanova T, Tangye SG, Newton R, Frank N, Hodge MR, Rolph MS, et al. T follicular helper cells express a distinctive transcriptional profile, reflecting their role as non-Th1/Th2 effector cells that provide help for B cells. J Immunol (2004) 173:68-78. doi:10.4049/jimmunol.173.1.68

27. Vogelzang A, McGuire HM, Yu D, Sprent J, Mackay CR, King C. A fundamental role for interleukin-21 in the generation of $\mathrm{T}$ follicular helper cells. Immunity (2008) 29:127-37. doi:10.1016/j.immuni.2008.06.001

28. He J, Tsai LM, Leong YA, Hu X, Ma CS, Chevalier N, et al. Circulating precursor CCR7lo.PD-1hi. CXCR5+. CD4+. T cells indicate Tfh cell activity and promote antibody responses upon antigen reexposure. Immunity (2013) 39:770-81. doi:10.1016/j.immuni.2013.09.007

29. Rivino L, Messi M, Jarrossay D, Lanzavecchia A, Sallusto F, Geginat J. Chemokine receptor expression identifies Pre-T helper Th.1, Pre-Th2, and nonpolarized cells among human CD4+ central memory T cells. J Exp Med (2004) 200:725-35. doi:10.1084/jem.20040774

30. Morita R, Schmitt N, Bentebibel SE, Ranganathan R, Bourdery L, Zurawski G, et al. Human blood CXCR5+.CD4+. T cells are counterparts of T follicular cells and contain specific subsets that differentially support antibody secretion. Immunity (2011) 34:108-21. doi:10.1016/j.immuni.2011.01.009
31. Bentebibel SE, Lopez S, Obermoser G, Schmitt N, Mueller C, Harrod C, et al. Induction of ICOS+CXCR3+CXCR5+ TH cells correlates with antibody responses to influenza vaccination. Sci Transl Med (2013) 5:176ra32. doi:10.1126/scitranslmed.3005191

32. Phetsouphanh $\mathrm{C}, \mathrm{Xu}$ Y, Zaunders J. CD4 T cells mediate both positive and negative regulation of the immune response to HIV infection: complex role of $\mathrm{T}$ follicular helper cells and regulatory $\mathrm{T}$ cells in pathogenesis. Front Immunol (2014) 5:681. doi:10.3389/fimmu.2014.00681

33. Iyer S, Gangandhara S, Victor B, Gomez R, Basu R, Hong J, et al. Co-delivery of envelope protein in alum with MVA vaccine induces CXCR3-biased CXCR5+ and CXCR5-CD4 T cell responses in rhesus macaques. JImmunol (2015) 195(3):994-1005. doi:10.4049/jimmunol.1500083

34. Vinuesa CG, Cook MC. Blood relatives of follicular helper T cells. Immunity (2011) 34:10-2. doi:10.1016/j.immuni.2011.01.006

35. Hong JJ, Amancha PK, Rogers K, Ansari AA, Villinger F. Spatial alterations between CD4+. T follicular helper, B, and CD8+. T cells during simian immunodeficiency virus infection: T/B cell homeostasis, activation, and potential mechanism for viral escape. J Immunol (2012) 188:3247-56. doi:10.4049/ jimmunol.1103138

36. Lindqvist $\mathrm{M}$, van Lunzen J, Soghoian DZ, Kuhl BD, Ranasinghe S, Kranias G, et al. Expansion of HIV-specific T follicular helper cells in chronic HIV infection. J Clin Invest (2012) 122:3271-80. doi:10.1172/JCI64314

37. Petrovas C, Yamamoto T, Gerner MY, Boswell KL, Wloka K, Smith EC, et al. CD4 T follicular helper cell dynamics during SIV infection. JClin Invest (2012) 122:3281-94. doi:10.1172/JCI63039

38. Cubas RA, Mudd JC, Savoye AL, Perreau M, van Grevenynghe J, Metcalf T, et al. Inadequate $\mathrm{T}$ follicular cell help impairs B cell immunity during HIV infection. Nat Med (2013) 19:494-9. doi:10.1038/nm.3109

39. Miles B, Miller SM, Folkvord JM, Kimball A, Chamanian M, Meditz AL, et al. Follicular regulatory $\mathrm{T}$ cells impair follicular T helper cells in HIV and SIV infection. Nat Commun (2015) 6:8608. doi:10.1038/ncomms9608

40. Chowdhury A, Del Rio Estrada PM, Tharp GK, Trible RP, Amara RR, Chahroudi A, et al. Decreased $\mathrm{T}$ follicular regulatory cell/T follicular helper cell TFH. in simian immunodeficiency virus-infected rhesus macaques may contribute to accumulation of TFH in chronic infection. J Immunol (2015) 195:3237-47. doi:10.4049/jimmunol.1502269

41. Boswell KL, Paris R, Boritz E, Ambrozak D, Yamamoto T, Darko S, et al. Loss of circulating CD4 T cells with B cell helper function during chronic HIV infection. PLoS Pathog (2014) 10:e1003853. doi:10.1371/journal.ppat.1003853

42. Chevalier N, Jarrossay D, Ho E, Avery DT, Ma CS, Yu D, et al. CXCR5 expressing human central memory CD4 T cells and their relevance for humoral immune responses. J Immunol (2011) 186:5556-68. doi:10.4049/jimmunol.1002828

43. Cubas R, van Grevenynghe J, Wills S, Kardava L, Santich BH, Buckner CM, et al. Reversible reprogramming of circulating memory $\mathrm{T}$ follicular helper cell function during chronic HIV infection. J Immunol (2015) 195:5625-36. doi:10.4049/jimmunol.1501524

44. Pallikkuth S, Parmigiani A, Silva SY, George VK, Fischl M, Pahwa R, et al Impaired peripheral blood T-follicular helper cell function in HIV-infected nonresponders to the $2009 \mathrm{H} 1 \mathrm{~N} 1 / 09$ vaccine. Blood (2012) 120:985-93. doi:10.1182/blood-2011-12-396648

45. Perreau M, Savoye AL, De Crignis E, Corpataux JM, Cubas R, Haddad EK, et al. Follicular helper T cells serve as the major CD4 T cell compartment for HIV-1 infection, replication, and production. J Exp Med (2013) 210(1):143-56. doi:10.1084/jem.20121932

46. Yi JS, Du M, Zajac AJ. A vital role for interleukin-21 in the control of a chronic viral infection. Science (2009) 324:1572-6. doi:10.1126/science.1175194

47. Elsaesser H, Sauer K, Brooks DG. IL-21 is required to control chronic viral infection. Science (2009) 324:1569-72. doi:10.1126/science.1174182

48. Yue FY, Lo C, Sakhdari A, Lee EY, Kovacs CM, Benko E, et al. HIV-specific IL-21 producing CD4+ T cells are induced in acute and chronic progressive HIV infection and are associated with relative viral control. J Immunol (2010) 185:498-506. doi:10.4049/jimmunol.0903915

49. Saez-Cirion A, Bacchus C, Hocqueloux L, Avettand-Fenoel V, Girault I, Lecuroux C, et al. Post-treatment HIV-1 controllers with a long-term virological remission after the interruption of early initiated antiretroviral therapy ANRS VISCONTI study. PLoS Pathog (2013) 9:e1003211. doi:10.1371/ journal.ppat.1003211

50. Moir S, Fauci AS. B cells in HIV infection and disease. Nat Rev Immunol (2009) 9:235-45. doi:10.1038/nri2524 
51. Fahey LM, Wilson EB, Elsaesser H, Fistonich CD, McGavern DB, Brooks DG. Viral persistence redirects CD4 T cell differentiation toward T follicular helper cells. J Exp Med (2011) 208:987-99. doi:10.1084/jem.20101773

52. Kwong PD, Mascola JR. Human antibodies that neutralize HIV-1: identification, structures, and B cell ontogenies. Immunity (2012) 37:412-25. doi:10.1016/j.immuni.2012.08.012

53. Pissani F, Streeck H. Emerging concepts on T follicular helper cell dynamics in HIV infection. Trends Immunol (2014) 35:278-86. doi:10.1016/j.it.2014.02.010

54. Cohen K, Altfeld M, Alter G, Stamatatos L. Early preservation of CXCR5+ PD-1+ helper T cells and B cell activation predict the breadth of neutralizing antibody responses in chronic HIV-1 infection. J Virol (2014) 88:13310-21. doi:10.1128/JVI.02186-14

55. Yamamoto T, Lynch RM, Gautam R, Matus-Nicodemos R, Schmidt SD, Boswell KL, et al. Quality and quantity of TFH cells are critical for broad antibody development in SHIVAD8 infection. Sci Transl Med (2015) 7:298ra120. doi:10.1126/scitranslmed.aab3964

56. Moody MA, Pedroza-Pacheco I, Vandergrift NA, Chui C, Lloyd KE, Parks R, et al. Immune perturbations in HIV-1-infected individuals who make broadly neutralizing antibodies. Sci Immunol (2016) 1:aag0851-0851. doi:10.1126/ sciimmunol.aag0851

57. Fletcher CV, Staskus K, Wietgrefe SW, Rothenberger M, Reilly C, Chipman JG, et al. Persistent HIV-1 replication is associated with lower antiretroviral drug concentrations in lymphatic tissues. Proc Natl Acad Sci U S A (2014) 111:2307-12. doi:10.1073/pnas.1318249111

58. Hufert FT, van Lunzen J, Janossy G, Bertram S, Schmitz J, Haller O, et al. Germinal centre CD4+ T cells are an important site of HIV replication in vivo. AIDS (1997) 11:849-57. doi:10.1097/00002030-199707000-00003

59. Kohler SL, Pham MN, Folkvord JM, Arends T, Miller SM, Miles B, et al. Germinal center T follicular helper cells are highly permissive to HIV-1 and alter their phenotype during virus replication. J Immunol (2016) 196:2711-22. doi:10.4049/jimmunol.1502174

60. Pallikkuth S, Sharkey M, Babic DZ, Gupta S, Stone GW, Fischl MA, et al. Peripheral T follicular helper cells are the major HIV reservoir within central memory CD4 $\mathrm{T}$ cells in peripheral blood from chronically HIV-infected individuals on combination antiretroviral therapy. J Virol (2016) 90:2718-28. doi:10.1128/JVI.02883-15

61. Fukazawa Y, Lum R, Okoye AA, Park H, Matsuda K, Bae JY, et al. B cell follicle sanctuary permits persistent productive simian immunodeficiency virus infection in elite controllers. Nat Med (2015) 21:132-9. doi:10.1038/ nm. 3781

62. Boritz EA, Darko S, Swaszek L, Wolf G, Wells D, Wu X, et al. Multiple origins of virus persistence during natural control of HIV infection. Cell (2016) 166:1004-15. doi:10.1016/j.cell.2016.06.039

63. Leong YA, Chen Y, Ong HS, Wu D, Man K, Deleage C, et al. CXCR5+ follicular cytotoxic T cells control viral infection in B cell follicles. Nat Immunol (2016) 17:1187-96. doi:10.1038/ni.3543

Conflict of Interest Statement: The authors declare that the research was conducted in the absence of any commercial or financial relationships that could be construed as a potential conflict of interest.

Copyright (c) 2017 Thornhill, Fidler, Klenerman, Frater and Phetsouphanh. This is an open-access article distributed under the terms of the Creative Commons Attribution License (CC BY). The use, distribution or reproduction in other forums is permitted, provided the original author(s) or licensor are credited and that the original publication in this journal is cited, in accordance with accepted academic practice. No use, distribution or reproduction is permitted which does not comply with these terms. 